\title{
DOCUMENTACIÓN NOTARIAL E HISTORIA DE LA SANIDAD
}

\section{MERCEDES GALLENT MARCO}

Universidad de Valencia

Pretendemos en el presente trabajo incidir en algunos aspectos relativos a la Historia de la Sanidad en el País Valenciano a partir de una tipología de fuentes - archivo notariales- (1) distintas a las que hasta ahora habiamos utilizado. Tales fuentes, todavía poco explotadas por los historiadores, aportan un material de primer orden para todo aquél que intente aproximarse a la problemática económica, religiosa, social, a la vida material, a las actitudes mentales de una época determinada. Así lo constata André Corvisier en su obra Sources et méthodes en histoire sociale cuando anota: «Aucun document ne parait plus ponctuel et personnel qu'une minute notariale, puisqu'elle concerne la vie et les intérêts d'un individu, d'une famille, tout au plus d'un groupe d'associés, en fixant les clauses d'un contrat, les termes d'un testament ou d'une donation, etc. En effet on rencontre essentiellement dans les minutiers, des documents de caractère familial: contrats de mariage, donations, testaments, partages de biens, inventaires après decès qui marquent de grandes étapes dans la vie. En outre, relatifs à des intérêts matériels, les minutiers contiennent un nombre très important d'actes de caractère économique: ventes et achats, emprunts, constituions de rentes, baux, constitutions de sociétés, etc.» (2)

Esta primera incursión en los archivos notariales queremos, pues, plantearla desde una perspectiva, diríamos «metodológica», intentando elaborar unas pautas que puedan servirnos en un futuro como posible «modelo» a seguir cuando estudiemos documentación notarial referente a temas relacionados con la sanidad o sus representanes. 
El valor intrínseco que esta tipología de fuentes tiene para nosotros estriba en que nos permite entrever determinados aspectos, tanto científicos como cotidianos o sociales, de los profesionales de la sanidad valenciana. Dada la variedad de información que ofrecen orientaremos su estudio y análisis a partir de las perspectivas siguientes:

- Posibilidad de un mayor conocimiento de los profesionales de la sanidad valencianos, facilitado su posterior tratamiento prosopográfico.

- Posibilidad de conocer las fuentes de formación o consulta que solía utilizar el profesional sanitario.

El contenido del documento objeto de este trabajo (3) se refiere al inventario post mortem que se hizo de los bienes del cirujano valenciano Rafael de Mena y de la venta posterior (almoneda) de los mismos, los heredados por su esposa, Na Johana, y pos su hijo, Rafael de Mena, presbitero, beneficiado y "domer" de la Catedral de Valencia, actuando su madre como procuradora. Procuraremos comprobar en él los puntos citados anteriormente.

1. Trataremos, respecto al primer interrogante, de reconstruir paulatinamente la trayectoria de una serie de personajes, barberos y cirujanos en nuestro caso, por su interés para el estudio de la sanidad valenciana bajomedieval porque, como bien sabemos, tenemos información acerca de las actuaciones de ciertos profesionales socialmente reconocidos y encumbrados que encontramos detentando un número elevado de cargos: «protofisics», «protocirurgians», "desospitadors», examinadores del municipio, médicos de hospitales... (4), pero muy poco o nada sabemos de aquellos otros profesionales anónimos sobre los que, en definitiva, recála una responsabilidad y ejercían un quehacer diario no siempre reconocido.

La enumeración de todos los barberos y cirujanos que aparecen en nuestro documento así lo demuestra, de hecho, los 16 barberos y cirujanos en él citados: «mestre» Rafael de Mena; Petro Portogués, "barbitonsoris Valencie»; Lope de Navarro, «barber e cirurgià»; «mestre» Johan Eximeneç; «barber e cirurgiá»; «mestre» Arnau Guascó, «barber»; Johan Moles «barber»; Ferrando Caseres, «barber»; Anthoni Alguaro, «barber»; Berthomeu Sebastià, "barber»; Johan Draçan, alias De Leyda; Simó Soriguera, "barber»; Johan d'Aragón, «barber»; Sebastià Tort y Nicholau Florentí, son prácticamente desconocidos para nosotros y hemos podido constatar que sólo cuatro de ellos desempeñaron una serie de cargos importantes y de prestigio:

- Rafael de Mena, el «protagonista» y al parecer el de más renombre. Se encuentra en 1458 actuando como testigo en la petición que los mayora- 
les de la cofradía de barberos y cirujanos dirigieron al gobernador de Valencia para poder reunirse libremente, donde declara:

"En Rafael de Mena, barber e cirurgià de la ciutat de València, testimonis qui jura, etc. E dix que ell stat majoral del dit offici e ensemps ab sos companyons del dit offici e almoyna se son ajustats en lo monestir de la Mercè, contractant fets e negocis sens convocar-li may official algú per lo Senyor Rey, e axi u ha vist practicar de XV anys que té obrador...» (5)

Además es majoral de la "confratria e almoyna dels barbers" junto con Johan Masco, Goçalbo Sanç y Jacme Ros en 1460 (6). Así mismo, en 1468, con motivo de ciertas remodelaciones que se hace en las constituciones del colegio, aparece como diputado del mismo (7). Fue elegido «conseller» de oficio, representante de los «barbers» en los años 1461, 1464 y 1467 (8) y nombrado «examinador dels cirurgians» por el Consell de la ciudad durante los años $1460,1466,1470,1472,1480$ (9).

- Johan Eximeneç, fue examinador de cirujanos en 1446 y "conseller» de oficio en 1448 (10).

- Simó Soriguera, es“conseller» de oficio en 1498 y 1506 (11).

- Anthoni Devot, se le nombró examinador de cirujanos en 1505 (12).

LLama la atención, en el tratamiento de estos personajes la confusión reflejada respecto a la titulación atribuida a estos profesionales, indicativa del desconocimiento general en esta sociedad sobre la gradación profesional, pues si bien encontramos correcta la distinción (por supuesto lenta y paulatina) entre «barber» y «cirurgià», o en su caso la utilización conjunta de ambos, nos llama la atención la utilización del término «mestre», que indica la adquisición del máximo grado académico en los estudios médicos (13).

2. Es útil, también, la información aportada por este documento para el reconocimiento de determinadas patologías, terapias y material quirúrgico, aunque no sea excesivamente explícito.

Reiteradamente se nos habla (en función de la utilización del instrumental) de «mal de lengua», 1; «mal de nas», 2; «mal de ses e del budell insà prop d'aquell», 3; «mal de cap», 4, 5, 9; «mal de cap e nafres», 6; «malalties o nafres de cap», 7, 10, 11, 12, 13; «malaltia de cap», 15; «mal de boqua», 22; «squinança»,56, indicios evidentemente vagos (cabria hablar de estomatitis, cefalalgias, amigdalitis, esquinancia, hemorroides...) pero referidos a dolencias susceptibles, como se constata, de un tratamiento quirúrgico: «levar squer- 
des de ossos per a levar malalties de cap», 16; «arrancar quexals», 20; cauterizar; «dar cauteris de fosch», 21; trepanar 8, 51 .

Se enumera, por otra parte, de forma prolija el instrumental propiamente dicho, aunque tropezamos con la dificultad de su reconocimiento desde el punto de vista técnico (14), consecuencia, como cabe suponer, de la ignorancia de quien hace el inventario. A pesar de todo podemos hacer la siguiente relación indicativa:

- Instrumentos de hierro sin especificar: «instruments de ferre", "ferros", uferros de cirurgia», 1, 2, 3, 4, 10, 15, 16, 20, 25, 50, 51, 52, 54, 56, 57, 64 .

- Instrumentos de hierro curvado: «Instruments de ferrer voltats» (¿separadores, forceps, cucharillas?), 6.

- «Ferros a manera de peus de grua», 26.

- «Caveguets de ferre» (a modo de «azadilla» de hierro, (¿lepra?), 5.

- «Lambroix de ferre», «ferros lambroxos» (hierros de rebajar, ¿gubia?), 7,55 .

- «Trepans" (trépanos), 8, 51.

- «Barrines de ferre» (taladro), «barrines ab ses virolles de ferrer» (taladros con sus abrazaderas de hierro), 9, 29, 30, 37.

- «Ferrer apellats gomietes» (doble hierro curvado utilizado por los herreros para engomar), 12.

- «Escarprets de ferre» (escalpelos), 11, 13.

- Varios tipos de tenazas: «mordaretes", «tenalletes de ferrer de barama neta», 17, 18, 57 .

- Pinzas: «guaffa per arrancar quexals», 19.

- Cauterios: «cauteris de ferre», en forma de dátil, de oliva: $21,32,33$, $34,58,50,60,61,62,63$.

- Instrumentos de latón: «Instruments de leuto voltat», 22, 27.

- «Martellets de ferre a modo de clau d'arpa», 23.

- Tijeras: "Tisoretes de ferrer a odo de tisores de baxador", 24.

- "Levador de ferre tallant» (instrumento utilizado en cirugía para destapar una herida, ¿erina?), 26. 
- Especulos: «speculum», 56, 65.

- Abrebocas, utilizados para poder manipular en la cura de ciertas enfermedades: «baoales», 53; «badall de cura de squinança», 56; «instruments de ferre per a tenir la boca ubertaw, 31.

- «Ferros apellats enginchs de passadors», (probablemente agujas de diversos tipos, lancetas) 66.

- Diversos estuches con los instrumentos propios del barbero: «stoig de barber", "stoig de cinta ab sos arreus», 41, 71.

- Instrumentos para quitar esquirlas de los huesos: «bec de ferrer a manera de bec de grua», «bec de grua», 38, 54 .

- Instrumento para hacer pildoras: «magdalió de diafarmacium», 28 (15).

3. Incidiremos, por último en el tercer punto que se refiere, como dijimos, a la oportunidad que nos brinda esta tipología documental para poder entrever cuales eran las fuentes de estudio y/o consulta utilizadas normalmente por los médicos, cirujanos y barberos.

Conocemos bien los libros «de texto" obligatorios, e incluso los que se podían utilizar voluntariamente en los estudiso de medicina, fijados por los estatutos de cada estudio general (16), pero desconocemos cuáles eran los usados por los cirujanos y barberos.

Esto no significa que tales profesionales carecieran totalmente de una formación «institucional», pues, como indica Cardoner i Planas, "Al segle XIV a la Corona d'Aragó, pero, hi hagueren molts cirurgians formats "cientificament" a Montpeller o Itàlia, llocs en els quals els qui volien ésser-ho estudiaven la part teòrica, mentre que per a la pràctica es valie de l'ensenyament privat, posant-se durant cinc anys al servei —com a berbers- d'un cirurgià vell, mitjançant uns típics contractes d'aprenentatge» (17).

Este colectivo, al igual que el valenciano, demostró siempre una creciente preocupación -evidenciada en los diversos estatutos de su gremio, y en su interés por la institucionalización oficial de la enseñanza de la Cirugíapor adquirir una formación cientifica teórica y práctica.

Recordemos al respecto, lo estipulado en los estatutos de 1433, donde se regulan los estudios y aprendizaje de quien desee ejercer la cirugía; el establecimiento en 1462, por la ciudad y a instancias de aquellos, de una lectura de cirugia, puesto que era necesario "quel.s barbers e cirurgians fossen abtes e scients e experts en l'art e pratica de cirurgia» y estaban dispuestos 
a «aprendre e desijosos de saber en la dita art si havia quil.s instruits e legis de la dita art de cirurgia, e en la dita ciutat hi hagués de bons fisichs e doctors abtes e competents a legirn; el privilegio concedido por Juan II en 1478 para disecar cadáveres, que les permite profundizar en el conocimiento del cuerpo humano, pues es importante «poder veure, saber, tractar e provehir per los ropis vulls aquelles cosses ocultes e amagades dins los cossos mundanals» $y$, por último, los capítulos de 1486, en los que se plasma la voluntad de barberos y cirujanos por conformar su «status» social como y delimitar lo relativo a su formación y ejercicio profesional, se alude también en el punto cuarto, con cierta ambigüedad a las materias que debían conocer y de las que tenían que examinarse, ante los mayorales del colegio primero y luego, si eran aprobados, ante los examinadores del municipio:

"E si en lo dit examen... serà atribat abte e suficient axí en la dita pràtica et in moribus et vita sia per los quatre mayorals que en aquell any seràn, presentat als quatre examinadors reals en la forma contenguda en lo primer capitol per la dita insigne ciutat elects, los quals quatre examinadors lo hajen e sien tenguts interrogar de la theorica axí com en la dita art de cirurgia se pertany. E si per aquell lo qui al dit examen serà admés per tots concordantment et nemine discrepante serà atrobat abte e suficient in scientia moribus et vita...» (18)

La práctica, como sabemos, debía ejercerse con un cirujano experto y estaba sujeta a unas reglas (19), pero desconocemos las materias y los textos objeto de estudio. (In scientia moribus et vita...) aunque podamos imaginarlo a partir de los libros registrados en el inventario dado que su dueño era un cirujano bastante conocido (20) lo que nos obliga a suponer tuviera una buena formacion.

En el texto se citan las siguientes obras:

- «Un libre de cirurgia... apellat lo Embranc», 43, 68.

- «Altre libre de cirurgia compost per mestre Bru», 44, 69.

- «Altre libre... apellat Tederic de cirurgia, compost per frare Tedericus», 45, 93.

- «Altre libre de cirurgia apellat Albuaquasis compost per mestre Rasis», 46, 94.

- «Altre libre apellat Guido», 47.

- «Hun libre apellat Anathomia Mundi», 49, 92. 
El libro de cirugía citado como lo Embranc creemos que se refiere a la obra de Lanfranchi de Melas (+ 1306) titulada Chirurgia Magna. Lanfranco, discipulo de Guillermo de Saliceto, se formó en el estudio general de Bolonia, donde éste impartía la enseñanza. Forzado a exiliarse por razones políticas marchó a París (hacia 1296), donde llevó «la brillante cirugía italiana del siglo XIII». Su obra más conocida, la Chrirurgia, es fruto «de una intensa actividad clínica y docente», erigiéndose como uno de los fundamentos de la cirugía francesa bajomedieval.

La gran difusión que tal obra tuvo nos la prueban las numerosas ediciones que de ella se han hecho, desde su traducción al francés en 1498 hasta la edición más reciente de Tabanelli en 1965. En la Corona de Aragón lo encontramos formando parte de los códices de la biblioteca del monasterio de Ripoll, junto con Tederico de Luca y Bru de Longoburgo, siendo traducido del latín al catalán en el siglo XIV por Corretger de Mallorca (21).

La "Cirugia composta per mestre Bru» pensamos que se trata de una de las obras Chirurgia Magna o Chirurgia Parva del cirujano italiano Bruno de Longoburgo, formado también en Bolonia, en la escuela (tal vez privada) de Hugo Borgognoni de Lucca. Sus obras, compuestas hacia 1252, fueron impresas hacia 1498 en Venecia y traducidas al catalán en el siglo XIV (22).

El «Tederc de cirurgia» parece ser la obra llamada Chirurgia, compuesta por Teoderico de Lucca (1206-1298), dominico y obispo de Cervia, formado al igual que Bruno, en la escuela de Hugo Borgognoni y con «autorización eclesiástica para ejercer sin lucro personal la cirugíam. Dicho libro, Tederico, lo dedicó a Andrés Albalat, obispo de Valencia, con el que trabó amistad durante su estancia en Roma. También fue traducido al catalán en el siglo XIV por Guillem Corretger (23).

El manual citado como «libre de cirurgia apellat Albuquasis compost per mestre Rasis» (cuando se cita de nuevo sólo se refiere al «libre apellat Abducasis"), quizás indiquen dos libros de autores diferentes encuadernados juntos que crearon confusión y son citados como uno solo.

El primero acaso se refiere a la obra del cirujano cordobés del siglo $X$, Abul-Quasim Al-Zaharawi, titulada Katib-al-Tasrif, compendio del saber médico de su tiempo y cuyo libro XXX, dedicado a la cirugía gozó de gran prestigio hasta el siglo XVIII. Su uso y difusión fue importante, como atestiguan las diversas traducciones que de él se hicieron. Según Cardoner i Planas: «l'obra d'Abu-I-Qasim - que conté la millor cirurgia escrita per cap autor àrab- fou traduïda de l'arab al llatí en el segle XIl per Gerard de Cremona a Toledo; 
en la segona meitat del XIII del l'àrab a l'hebreu per Shem Tob Ibn Isaac a Marsella, i al començament del segle XIV fou traslldada de l'àrab al català pel jueu Jafuda Bonsenyor» (24).

La referencia a Rases quizás indique otro autor musulmán, el persa Rhazes (Abu Bakr Muhammad Ben Zakariyya al-Rhazi, 865-932), en palabras de Lain Entralgo «primera gran figura de la medicina árabe». Su obra más famosa fue la conocida como Kitab-al-Mansur, manual dividido en diez partes y en el que se trata de toda la teoría y la práctica de la medicina. Traducido también por Gerardo de Cremona al latín después de 1270, y en el siglo XIV al catalán, se convirtió, en especial el libro IX, en uno de los textos fundamentales para la enseñanza de la medicina (25).

El libro citado como «Guido", debe referirse sin duda a Guy de Chauliac ( + 1368), médico formado en Montpelier, donde se doctora (1325), y en Bolonia, cuya obra Chirurgia Magna, traducida en el siglo XV del latín al catalán por Bernat de Casaldovol y Jeroni Masnoves «es el tratado de cirugía más influyente desde la Baja Edad Media hasta el siglo XV|» (26).

Del último libro registrado sólo conocemos el título: «Anathomia Mundi», pero no aporta referencia o indicio alguno respecto a su autor; aventuramos, no obstante que se trata de la obra de Mondino de Luzzi $(+1327)$, Anathomia compuesta en 1316.

Mondino, alumno y posteriormente profesor del estudio boloñés, ha sido considerado «como la máxima figura del saber anatómico medieval» siendo su obra «el texto básico para la enseñanza de la "medicina theórica" en muchas universidades hasta la publicación de la Fábrica de Vesalio, y en algunas hasta más tardè lo que explica las sucesivas reimpresiones que de él se hicieron a partir de la aparición de la «editio princeps» en 1478 (27).

Estos datos confirman, pues, el evidente influjo de la medicina italiana en los profesionales valencianos (28) y un nivel «cientifico» bastante elevado, acorde con la opinión expuesta por Cardoner y Planas cuando, aludiendo a la formación de los cirujanos de la Corona de Aragón, dice «Per a documentarse... es feren traduccions vernacles dels llibres d'Abu-I-Quasim... així foren incorporats a la cultura quirurgica catalano-aragonesa, Roger de Salern, Bru de Longoburgo, Tederic de Lucca, Guillem de Saliceto i Lanfranc...», «A ells s'hi afegí -anota más adelante- però el text acabat el 1363 a Avinyó pel francès Guy de Chauliac...» (29)

Es evidente, por consiguiente, la riqueza informativa de este tipo de documentación. Se impone, lógicamente, una búsqueda exhaustiva de docu- 
mentos similares para que, analizados y parangonados, podamos consolidar los datos aquí localizados, incorporar otros nuevos y, finalmente, configurar una visión más completa de los aspectos sanitarios de Valencia medieval y, muy especialmente, un conocimiento de lo más exacto posible de la formación y mentalidad de los profesionales.

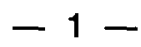

1485, enero, 13. Valencia

Inventario de los bienes de mestre Rafael de Mena.

A. P. P. V., Joan Cabanes

\section{Die iovis XIII ianuarii anno a Nativitate Domini M. ${ }^{\circ} \mathrm{CCCCLXXXV}$}

Consecutive vero dicta die iovis intitulata XIII ianuarii anno iamdicto a $\mathrm{Na}$ tivitate Domini $M .{ }^{\circ} \mathrm{CCCCLXXX}$ quinto, lo dit honorable En Johan Francesc en lo dit de procurador de la dita $\mathrm{Na}$ Johana, muller e hereva del dit mestre Raffahel de Mena, continuant lo inventari dels béns del dit mestre Rafael e de la herència dessus dita feu inventari dels bens següents atrobats dins dos caxonets cascú per si apel.lats caxonets de la ferramenta, ferros de cirurgia, los quals són los següents atrobats en los dits caxonets:

\section{$-1-$}

[1]. PRIMO: hun instrument de ferre per a curar malaltia de mal de lengua.

[2]. ITEM: altre instrument de ferre per a curar malaltia de mal de nas.

[3]. ITEM: altre instrument de ferre per a curar mal de ses e del budell insá prop de aquell.

[4]. ITEM: dos instruments de ferre a manera de ferrer per a curar mal de cap.

[5]. ITEM: set caveguets de ferre, instruments per a curar mal de cap.

[6]. ITEM: XII instruments de ferre voltats per a curar malalties de cap e nafres.

[7]. ITEM: hun lambroix de ferre per a curar malalties o nafres de cap.

[8]. ITEM: dos trepans de ferre per a obrar en mal de cap o nafres de cap.

[9]. ITEM: dos barrines de ferre per a obrar en mal de cap.

[10]. ITEM: dos instruments de ferre de dos peces cascú per a obrar en malalties o nafres de cap.

[11]. ITEM: dos scarpets de ferre per a curar malalties o nafres de cap.

[12]. ITEM: tres ferres apellats gomietes per a curar malalties o nafres de cap.

[13]. ITEM: dos starpres de ferre per a curar malalties o nafres de cap.//

[14]. ITEM: tres gomietes de ferre per a curar malalties de mal de cap.

[15]. ITEM: dos ferros per a curar malaltia de cap. 
[16]. ITEM: tres ferres a manera de peus de grua per a levar squerdes de ossos per a curar malalties de cap.

[17]. ITEM: dos mordacetes de ferre per a curar malalties en diverses parts.

[18]. ITEM: quatre tenalletes de ferre, totes de barma neta, per a obrar e curar en diverses malalties.

[19]. ITEM: una guaffa per arrancar quexals ab dos peus de ferre a modo de peus de porch.

[20]. ITEM: instruments de ferre per arrancar rahels de quexals.

[21]. ITEM: cauteriets de ferre per a dar cauteris de foch.

[22]. ITEM: hun instrument de leutó daurat voltat per a curar mal de boqua.

[23]. ITEM: hun martellet de ferre a modo de clau de arpa.

[24]. ITEM: unes tisoretes de ferre a modo de tisores de baxador.

[25]. ITEM: hun instrument de ferre per arrancar quexals.

[26]. ITEM: hun levador de ferre tallant.

[27]. ITEM: hun instrument de ferre tallant.

[28]. ITEM: hun magdalió de diafarmacium.

$$
-2-
$$

ITEM: l'altre caxonet de fusta foren atrobats los ferros següents:.

[29]. ITEM: tres barrines de ferre totes per a traure ferros de passadors dels cossos dels hòmens.

[30]. ITEM: dos altres barrines ab ses birolles totes de ferre per a traure passadors dels cossos dels hòmens.

[31]. ITEM: hun instrument de ferre per a fer tenir la boca uberta per a curar lo mal que es en la boca.

[32]. ITEM: XXIII cautiris instruments de ferre dells punials e dels coltellades per a obrar e curar diverses malalties.

[33]. ITEM: dos cautiris de ferres per a curar de grans mals.

[34]. ITEM: tres cautiris de ferre per a curar grans mals.

[35]. ITEM: tres cautiris redons per a curar de grans mals.

[36]. ITEM: quatre cautiris de ferre a manera de oliva.//

[37]. ITEM: una barrina tota de ferre ab nou altres peces de ferre per obrar en cures de diverses malalties.

[38]. ITEM: un bec de ferre a manera de boc de grua per a certa cura.

[39]. ITEM: hun instrument de ferre per a arrancar [...] o passados dels cossos dels hòmens.

[40]. ITEM: sis scalfados de leutó per a portar aygua calenta.

[41]. ITEM: hun stoig de barber per a portar en la cuita ab ses rahos e altres ferres dins guarnits alguns los caps de argent e al sol del stoig guarnit d'argent.

[42]. ITEM: una pedra verda per affilar rahos ab lo cap fornit e guarnit d'argent. 
Et hec sunt bona que ad presenti se nos inveni in domo et bonis dicti deffuncti et herentie precontente parte protestor tamen quod si aliqua bona invenro seu invenire potero. In bonis dicti deffuncti quod alia possum inventariare et in presenti inventario continuare eseti de novo aliud inventarium facere presenti inventario in aliquo non obsente et quo tempus michi nomine quo supra e dicti principali mee quo modo. Presentibus pro testibus Petro Portogues et Lope Navarro barbitonsores Valentie civibus testibus ad predicta vocatis et assumptis.

Continuatione. Dicta die iovis XIII ianuarii

Proinde vero die predictum intitulata tredecima mensis ianuarii anno iamdicto a Nativitate Domini $M .{ }^{\circ} C C C C L X X X V .{ }^{\circ}$, lo dit honorable En Johan Francesc el dit nom continuant lo dit inventari dels béns del dit mestre Rafahel de Mena e de la herència dessus dita feu inventari dels béns del dit mestre Rafahel de Mena o de la dita herència, los quals són los següents:

[43].- PRIMO: hun libre de cirurgia-scrit en paper liguat ab post e hun-guafet e dos scudets illuminats ab ses rubriques vermelles cabletrat de letres blaves e vermelles platonat ab platons, cubert de aluda, apellat to Emfranc.

[44]. ITEM: altre libre de cirurgia compost per mestre Bru, scrit tot en paper ab cubertes de paper engrutat e cabletrat e rubricat de vermelló./I

[45]. ITEM: altre libre en paper scrit apellat Tederic, de cirurgia, compost per frare Tedericus, rubricat de vermello ab cubertes de fust cubertes de aluda ab dos guafets e sos scudets.

[46]. ITEM: altre libre de cirurgia apellat Albuquasis compost per mestre Rasis tot en paper ab cubertes de fusta rubricat de vermelló.

[47]. ITEM: altre libre apellat Guido.

[48]. ITEM: hunes ores en perguami ab diversos officis ab cubertes de fusta e cubertes damunt la fusta de vellut, cerrat ab guaffet d'argent e son scudet d'argent.

[49]. ITEM: hun libre apellat Anathomia Mundi.//

Die iovis XIII ianuarii anno a Nativitate Domini M. ${ }^{\circ} \mathrm{CCCCLXXX}$ quinto

Deinde vero die iovis intitulata XIII ianuarii anno iamdicto a Nativitate Domini $M .{ }^{\circ} C C C C L X X X$ quinto. Lo dit mestre Johan Francesc el dit nom de procurador de la dita Na Joahana, muller e hereva del dit mestre Raffahel de Mena quondam continuant la dita almoneda feta primerament convocatió e aldana per lo corredor de la confratria dels barbers, anant per la ciutat dema- 
nant aquells que fossen per al depres dinar a la casa del dit mestre Raffahel hon se faria almoneda de la ferramenta necessària de cirurgia com axí sia cosa acostumada e útil per què de les coses e robes e ferros pertanyents a cirurgia se. $n$ haria major a millor preu o preus que no faria si no.s feya la dita convocació lo deprés dinar en la dita casa del dit deffunt migancant Anthoní Aznar alias ferrer corredor de coll en publich encant presents molts e diversos barbers e cirurgians legitimes subastacions precedents feu vendes dels bés deius scrits per los preus en les persones infrasegüents:.

[50]. PRIMO: huit ferros de cirurgia per a curar de diversos mals, lo hu dels quals ferros se nomena bec de grua, an Anthoni Devot, barber e cirurgià, per I s. X d.

[51]. ITEM: set ferros per a curar de cirurgia, lo hun dels quals se apella barrina e los altres trepans, a mestre Johan Eximeneç, barber e cirurgià, per VIIII d.

[52]. ITEM: tretze ferros per a curar mals de cap a mestre Arnau Guastó, barber e cirurgià, per I s. VId.

[53]. ITEM: hun artifici de coure daurat apellat badalets per a curar a malaltia de boca, a mestre Pere Porthogues, barber e cirurgià, per I s. VII d.

[54]. ITEM: cinc ferros entre los quals hi havia hun bec de grua el altres ferres per a curar mals de cap e de boca, a mestre Lope Navarro, barber e cirurgià, per III s. Vld.

[55]. ITEM: nou ferros lambroxos e altres ferros per a cures de caps an Johan Moles, barber, per VIIII d.

[56]. ITEM: cinch ferros, lo hun dels quals ha nom speculum, e hun badall de cura de squinança, e hun trepan per a curar de diverses malalties al dit en Johan Molés, barber, per II s. III d.

[57]. ITEM: XII ferros de cirurgia entre los quals hi havia unes tenalles e altres ferres per a cura de mal de quexals, a mestre Arnau lo Guastón, barber e cirurgià, per II s./I

[58]. ITEM: cinch ferros, hun cautiri e les altres [...] pans per a cures de caps, a mestre Johan Eximeneç, barber e cirurgià, per VI s. III d.

[59]. ITEM: tres cautiris de ferro per a donar foch en nafres e altres malalties, a mestre Pere Porthogues, barber e cirurgià, per II $\mathrm{s}$.

[60]. ITEM: set peces de ferros apellats cautiris per a obrar diverses malalties, a mestre Arnau, barber, per II $s$.

[61]. ITEM: cinch ferros apellats cautiris a mestre Johan Eximeno, barber cirurgià, per $V \mathrm{~s}$.

[62]. ITEM: dos ferros cautiris per a curar malalties d'ossos an mestre Pere Portogues, barber e cirurgià, per VI d. 
[63]. ITEM: XIIII ferros apellats cautiris per a diversos cures al dit en Johan Molés, per I s. VI d.

[64]. ITEM: nou ferros per a curar diverses malalties, en temps ab lo caxonet de pi hon stavan los dits ferros e altres ferros an Johan Eximeno, barber e cirurgià, per II s. VI d.

[65]. ITEM: hun ferro apellat speculum, a mestre Ferrando Caseres, barber, per VI d.

[66]. ITEM: dos ferros apellats enginchs de passadors.

[67]. ITEM: hun scalfador de leutó per a portar aygua calenta.

[68]. ITEM: hun libre apellat el Enfranch en paper ab cuberta de fusta cubert de aluda ab guafets e escudets, a mestre Pere el Portogues, per XXXXIIII s.

[69]. ITEM: hun altre libre apellat lo Bru en paper ab cubertes de paper engrutat an Antoni Alguaro, barber, per IIII s. VI d.

[70]. ITEM: hun caxonet hon staven los dits ferros al dit en Johan Moles, barber, per VIIII s.

[71]. ITEM: hun stoig de cinta ab sos arreus de rahos e altres arreus, alguns guarnits de argent e al sol guarnit de argent, an Berthomeu Sebastià, barber, per XV s.

Suma universal dels preus procehits de la dita almo // neda e vendes dessús dites dels dits béns, noranta sous e cinch diners, dels quals deduilts hun sou e [...] diners que pagua lo dit mestre Johan Francesc el dia [...] al corredor qui feu la aldana per València quant a les cases dels barbers convocant aquelles e notifficant-los que fossen a la almoneda fahedora lo deprés dinar a la casa hon lo dit mestre Rafahel en vida sua stava e habitava hon farien almoneda e vendes dels béns del dit mestre Rafael e senyaladament dels libres e ferres e instruments pertanyents a cirurgia. Item, dona e pagua lo dit mestre Johan al corredor qui subastà e feu les dites vendes dos sous, les quals despeses foren tres sous VI diners. Restaren nets en poder del dit mestre Johan Francesc noranta quatre sous e once diners

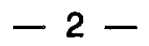

$$
\text { 1485, enero, 18. Valencia }
$$

Procura de Rafael de Mena, «domer» y beneficiado de la Catedral de Valencia, en favor de su madre Na Johana, para que pueda subastar los bienes legados a éste por su padre.
A. P. P. V., Joan Cabanes

Rafahel de Mena presbiter domerius sedis Valentie et benefficiatus in dicta sede de benefficio annexo dicte domerie et in predicta sede in statuto sub 
invocatione Sancti Spiritui revocando sive infamia quoscumque procuratores meos per me actenus sive constitutos. Scienter et gratis, facio, constituo et ordino procuratricem meam certam specialem et ad subscripta generalem ut in rem vestram propiam vos honorabile domnam Johanam matrem meam, uxorem honorabilis magistri Rafahelis de Mena cirurgiti et barbitonsoris Valentie civis, absentem vel ut presentem videlicet ad petendum, habendum, exhigendum, recipiendum et recuperandum loco mee et pro me quasvis res, raubas $[. .$.$] mutua debita deposita comandas acciperunt quantitates et alia$ quamvis bona que michi debentum et debebuntur per quantum quam personas universitates et collegia tam cum cartis et scripturis publicus vel privatus quam sine apud. Et de hiis que habueritis, exhigeretis, receperitis et recuperavitis ac // dicta dona Na johana, muller e hereva de mestre Rafael de Mena, cirurgià e barber de la dita ciutat, segons de la dita procura ab carta regunda per lo notari deins crit migançant Johan Centelles, corredor públic de coll de la dita ciutat dels béns scrits a les persones e per los preus infrasegüents:

Suma universal dels preus procehits de la dita almo // neda e vendes dessús dites dels dits béns, noranta sous e cinch diners, dels quals deduits hun sou e [...] diners que pagua lo dit mestre Johan Francesc el dia [...] al corredor qui feu la aldana per València quant a les cases dels barbers convocant aquelles e notifficant-los que fossen a la almoneda fahedora lo deprés dinar a la casa hon lo dit mestre Rafahel envida sua stava e habitava hon farien almoneda e vendes dels béns del dit mestre Rafael e senyaladament dels libres e ferres e instruments pertanyents a cirurgia. Item, dona e pagua lo dit mestre Johan al corredor qui subastà e feu les dites vendes dos sous, les quals despeses foren tres sous VI diners. Restaren nets en poder del dit mestre Johan Francesc noranta quatre sous e once diners

\section{$-2-$}

\section{5, enero, 18. Valencia}

Procura de Rafael de Mena, "domer" y beneficiado de la Catedral de Valencia, en favor de su madre Na Johana, para que pueda subastar los bienes legados a éste por su padre.

\section{A. P. P. V., Joan Cabanes}

Rafahel de Mena presbiter domerius sedis Valentie et benefficiatus in dicta sede de benefficio annexo dicte domerie et in predicta sede in statuto sub invocatione Sancti Spiritui revocando sive infamia quoscumque procuratores meos per me actenus sive constitutos. Scienter et gratis, facio, constituo et ordino procuratricem meam certam specialem et ad subscripta generalem ut 
in rem vestram propiam vos honorabile domnam Johanam matrem meam, uxorem honorabilis magistri Rafahelis de Mena cirurgiti et barbitonsoris Valentie civis, absentem vel ut presentem videlicet ad petendum, habendum, exhigendum, recipiendum et recuperandum loco mee et pro me quasvis res, raubas [...] mutua debita deposita comandas acciperunt quantitates et alia quamvis bona que michi debentum et debebuntur per quantum quam personas universitates et collegia tam cum cartis et scripturis publicus vel privatus quam sine apud. Et de hiis que habueritis, exhigeretis, receperitis et recuperavitis ac // dicta dona Na johana, muller e hereva de mestre Rafael de Mena, cirurgià e barber de la dita ciutat, segons de la dita procura ab carta regunda per lo notari deins crit migançant Johan Centelles, corredor públic de coll de la dita ciutat dels béns scrits a les persones e per los preus infrasegüents: [72]. PRIMO: una cadira de pi vellaja sotil antigua an Johan Molés, barber, per I s. VId.

[73]. ITEM: un baci de leutó sancer e bo al dit Moles, per VIII s. VIIId.

[74]. ITEM: altre baci de leutó sancer e bo al dit Molés, per VIII s. VIld.

[75]. ITEM: altre baci bo de leutó, emperò chich, al dit en Johan Moles, per $\mathrm{VI} d$.

[76]. ITEM: altre baci de leutó bo e sancer a ell mateix, per $\mathrm{VI} d$.

[77]. ITEM: altre baci de leutó bo e sancer am Johan Dracan, alias de Leyda, per VI s.

[78]. ITEM: hun scalfador de leutó bo e sancer an Simó Soriguera, barber, per XV s. VId.

[79]. ITEM: hum altre scalfador de leutó e bo e sancer al dit en Simó Soriguera, per XV s. VId.

[80]. ITEM: altre scalfador de leutó bo e sancer al dit en Johan Moles, per XV S. VId.

[81]. ITEM: altre scalfador de leutó bo e sancer al dit En Johan Molés, per $X V$ s. XVd.

[82]. ITEM: altre scalfador bo de leutó e sancer al dit En Johan Moles per obs de Sebastià Tort, barber, per XIIII s.

[83]. ITEM: altre scalfador de leutó, al costat hun poch trencat, an Johan Moles, per VII s. VIIIs.

[84]. ITEM: tres vergues de ferre per a tenir les calderetes al valor dels caps ab lo rajador al dit Moles, per III s.

[85]. ITEM: hun scalfador de caps de haram ab sos peus de ferre al dit En Moles, per XXIIII s. IId.

[86]. ITEM: hun ferre per a tenir los bacins al dit En Molés, per II $s$.

[87]. ITEM: un poal de coure b sa cadeneta de ferre per a poar aygua del pou al dit En Molés, per VI s. 
[88]. ITEM: un spill gran de porta ja trencat al dit En Molés, per II s.

[89]. ITEM: dos ferros de guins per a traure sagetes dels cossos dels hómens an Johan Francesc, per VI s.

[90]. ITEM: quatre altres ferros semblants als dessús dits, al dit En Johan Francesc, per IIII s.

[91]. ITEM: tres cadires sotils de pi de barber al dit En Moles, per [...].

[92]. ITEM: hun libre apellat Anathomia, scrit en paper ab cubertes de post an Johan d'Aragón, barber, per VII s.

[93]. ITEM: altre libre apellat Tederich, scrit en paper ab cubertes de fust, al dit En Molés, per VIs.

[94]. ITEM: altre libre apellat Abducasis, scrit en paper, al dit En Johan Franch, per $\mathrm{VI} d$.

[95]. ITEM: cinch tovalloles de lli trepades als caps al dit En Molés, per VIII s.

[96]. ITEM: una cortina de fil ab listes grogues per tenir en les barres a la porta, al dit Molés, per XII s.

[97]. ITEM: una cuberta de coxí sotil de lli ab hun trocet de drap sotil an $\mathrm{Ni}$ cholau Florenti, per I s.

[98]. ITEM: hun banc de pi sotil e ja vell ab tres banquets sotils per a tenir als peus dels que affayten, al dit En Moles, per I s.

Suma universal dels preus procehits de la dita almoneda dohents quaranta quatre sous sis diners, dels quals pagua al corredor de la dita almoneda III sous IIII. Item al lit on stava lo dit corredor ab la dita roba dos diners. De la resta pagua a Miquel Ortiz de València la dita herència XIIII lliures II sous VI diners. Restants dels dits preus per rahó de certa quantitat que li era dguda per lo dit mestre Rafahel e per ella dels quals lo dit Ortiz en lo dia de huy li.n ha fermat àpoca. 


\section{NOTAS}

(1) RODRIGO PERTEGAS, J. (1926) «Lo que hi ha en los protocols notarials de la epoca foral», Rev. de Cultura valenciana, p. 12-16. MATEU Y LLOPIS, F. (1956) "Notas sobre los archivos eclesiásticos y de protocolos del Reino de Valencia" Rev. de Árchivos, Bibliotecas y Museos, $L X I I, 3, p, 669-737$ (El catálogo completo del A. P. P. V. se está realizando en la actualidad). RODRIGUEZ TRONCOSO, R. (1986) Inventario de fondos notariales: Archivo del Reino de Valencia, Ed. Generalitat València-Conselleria de Cultura, col. Arxius Valencians 4, Valencia. DUALDE SERRANO. 147.

(2) CORVISIER, A. (1980) Sources et Méthodes en Histoire Sociale, Ed. SEDES, París, pág.

(3) A. P. P. V., Joan Cabanes (1485). Debo agradecer a mi compañero Vicente Pons Alós el haberme proporcionado desinteresadamente este documento.

(4) Cfr. GALLENT MARCO, M. (1980) La asistencia sanitaria en Valencia (1400-1512), 2 vols., tesis doctoral, inédita, vol. I, pág. 377-391; vol. II, p. 9-16, 38-66.

(5) GALLENT MARCO, M. (1980) Vol. II, p. 219-20; (1985) «El gremi de cirurgians de València: procés de constitució (1310-1499), Afers. fulls de recerca i pensament, 2, p. 249-269, cfr. p. 260.

(6) GALLENT MARCO, M. (1980), vol. II, p. 249-250; (1985) p. 260-61.

(7) GALLENT MARCO, M. (1985), p. 261-262.

(8) GALLENT MARCO, M. (1980), vol. II, p. 56-66.

(9) GALLENT MARCO, M. (1980), vol. II, p. 38-55.

(10) Cfr. notas 8 y 9.

(11) Cfr. nota 8.

(12) Cfr. nota 9

(13) DULIEU, L. (1975) La Médecine a Montpeller, vol. I, Le Moyen Age, Ed. Les Press Universelles, Avignon, p. 40-49. CARDONER I PLANAS, A. (1973), História de la Medicina a la Corona d'Aragó (1162-1479), Ed. Scientia, Barcelona. Este autor refiriéndose a los estatutos del Estudio General de Perpiñán promulgados entre 1380-1390, y a los que califica como sla codificació més clara de l'ensenyament de la medicina a la Corona d'Aragón al tratar sobre los estudios de medicina anota: «Es podien aconseguir tres graus: batxiller, llicenciat i mestre... Si es volia tenir el títol de "Mestre" -equivalent al de "Doctor" dels juristes-es sotmetia al llicenciat a un nou examen..." p. 83.

(14) A fin de poder reconocer en lo posible algún instrumental hemos consultado ALCOVER, A.; MOLL, F. de B. (1927-1962), Diccionari català-valencià-balear, Ed. Palma de Mallorca y CORACHAN M. (dir) (1936) Diccionari de Medicina, Ed. Salvat, Barcelona. Asi mismo es imprescindible la obra de GURTL, E. (1898), Geschichte der Chirurgie, Ed. Hirschwald, Berlín, a la que por causas ajenas a nuestra voluntad no hemos podido tener acceso. 
(15) Magdalio (magdaleon), pasta medicinal en forma de cilindro, ALCOVER-MOLL (19271962), vol. 7. p. 117. Consideramos que el texto indica a través de una expresión metonímica (contenido por el continente) el instrumento utilizado para hacer píldoras o «pindoler".

(16) CARDONER I PLANAS, A. (1973) Estatuts de Montpeller de 1309 y 1340, págs. 7375; Perpinyà, p. 83.

(17) CARDONER I PLANAS, A. (1973) p. 101.

(18) GALLENT MARCO, M. (1985) p. 253-268.

(19) Cfr. nota 19.

(20) Cfr. notas $5,6,7,8$ y 9 .

(21) LAIN ENTRALGO, F. (dir) (1972) Historia Universal de la Medicina, vol. III, Edad Media, Ed. Salvat, Barcelona, pág. 320-324; (1978) Historia de la Medicina, Ed. Salvat, Barcelona, p. 206. CARDONER I PLANAS. A. (1973), p. 30, 42. p. 42.

(22) LAIN ENTRALGO, P. (dir) (1972), p. 317, 319-320. CARDONES I PLANAS, A. (1973),

(23) LAIN ENTRALGO, F. (dir) (1972), p. 317-319; (1978) p. 205-206, 233-234, 236, CARDONES I PLANAS, A. (1973) p. 42. GARCÍA BALLESTER, L. (1969) «Aproximación a la Historia Social de la Medicina Española, VIII, Salamanca, págs. 45-78, pág. 53-62.

(24) LAIN ENTRALGO, P. (dir) (1972) p. 90-91; (1978), p. 159-160, 177, 190. CARDONES I PLANAS, A. (1973), p. 38, 164. VERNET, J. (1978), La cultura hispanoárabe en Oriente y Occidente, Ed. Ariel, Barcelona, p. 161-164.

(25) LAIN ENTRALGO, P. (dir) (1972) p. 92; (1978), p. 158-160, 169, 198, 235. CARDONES I PLANAS (1973), p. 38, 41.

(26) LAIN ENTRALGO, P. (dir) (1972), p. 305, 330-333; (1978), p. 209. CARDONER I PLANAS, A. (1973), p. 43.

(27) LAIN ENTRALGO, P. (dir) (1972), p. 300; (1978), p. 220.

(28) GARCIA BALLESTER, L. (1966-67), "Tres bibliotecas médicas en la Valencia del siglo XV», Asclepio, 18-19, p. 383-405; (1969) p. 73-75. BERGER, Ph. (1981), «La lecture a Valence de 1474-1560 (Evolution des comportements en fonction de milleur sociaux)", en Llibre et lecture en Espagne et en France sous l'Ancien Regine, Coloquio de la Casa de Velázquez, ed. A. D. P. F. París, p. 97-107.

(29) CARDONER I PLANAS, A. (1973), p. 168, 172. 